\title{
COMMENTARY
}

\section{Murine Double Minute-2 Links Cell Cycle Control and Inflammation in Homeostasis and Disease}

Martrez Ebrahim and Hans-Joachim Anders

From the Medizinische Klinik und Poliklinik IV, Klinikum der Universität München, LMU München, München, Germany

Murine double minute (MDM)-2 is a cytoplasmic protein well described to counteract the biological activity of p53 (ie, the central regulator of the cell cycle) in a yingyang-like manner. ${ }^{1,2}$ Although $\mathrm{p} 53$ inhibits cell cycle entry and ensures cell cycle arrest at the $\mathrm{G}_{1}$ or $\mathrm{G}_{2} / \mathrm{M}$ check points, MDM2 is rather a mitogenic factor. Indeed, in tumors, somatic loss-of-function mutations of the P53 gene promote tumor growth in an MDM2-dependent manner. ${ }^{3}$ Vice versa, gain-of-function mutations in the MDM2 gene have the same effect. ${ }^{3}$ MDM2 elicits this well-described p53 antagonistic biological effect in various ways (eg, ubiquitination, which directs $\mathrm{p} 53$ toward proteasomal degradation and nuclear export of p53 to the cytoplasm; and inhibition of the transcriptional activity of $\mathrm{p} 53$ by binding directly to the $\mathrm{N}$-terminal transactivation domain of p53) (Figure 1). In addition, through the negative feedback loop, p53 activation up-regulates MDM2 mRNA expression, which, in turn, leads to p53 degradation. ${ }^{4-7}$ However, in recent years, MDM2 was found to have additional p53-independent effects (Figure 1).

In the current issue of The American Journal of Pathology, Fan et $\mathrm{al}^{8}$ report on an anti-inflammatory role of MDM2 in the context of intraocular inflammation. They generated a retinal pigment epithelium cell line with an NF- $\kappa \mathrm{B}$ luciferase reporter and found that Nutlin-3, a wellcharacterized MDM2 inhibitor, blocked lipopolysaccharide (LPS)-induced NF- $\kappa \mathrm{B}$ activation. A deeper analysis revealed that MDM2 is required for the translocation of NF$\kappa \mathrm{B}$ from the cytosol to the nucleus, a process necessary for $\mathrm{NF}-\kappa \mathrm{B}$ binding to its promoter sequences and the induction of NF- $\kappa \mathrm{B}$ target genes. Interestingly, these effects were independent of $\mathrm{p} 53$, as proved by specific knockdown with p53 siRNA. To validate this proinflammatory NF$\kappa \mathrm{B}$-agonistic role of MDM2 in vivo, the authors used a model of LPS-induced ocular inflammation. The i.p. or intravitreal injection of the MDM2 inhibitor Nutlin-3 reduced NF- $\kappa \mathrm{B}$ activation in both the retina and the choroid, with a combination of both injection routes having the strongest effect. Again, this effect was p53 independent because Nutlin-3 elicited the same protective effect in p53deficient mice exposed to intraocular LPS injection. No negative effects of even supratherapeutic doses of Nutlin-3 on the retina or lenses were observed. The authors concluded that MDM2 inhibition with Nutlin-3 may be a valuable nonsteroidal way of reducing ocular inflammation in the anterior and posterior chambers of the eye, which may help to avoid the ocular (and systemic) adverse effects related to the use of steroids in this context (eg, in chronic uveitis).

This proinflammatory NF- $\kappa \mathrm{B}-$ agonistic effect of MDM2 was first described in 2009. Liu et $\mathrm{al}^{9}$ reported that MDM2 facilitates NF- $\kappa \mathrm{B}$ binding to its specific promotor-binding sites on NF- $\kappa \mathrm{B}$ target genes (Figure 1). They demonstrated that inflammatory cells treated with Nutlin-3a induced less apoptosis as well as decreased NF- $\kappa$ B DNA binding activity in nuclear extracts in response to LPS stimulation, without alterations in toll-like receptor 4 signaling pathways. Furthermore, p53-deficient mice showed an increased severity of LPS-induced acute lung injury, whereas pretreating $p 53$-deficient mice with Nutlin3a attenuated this inflammatory response. ${ }^{9}$ Subsequently, this observation was confirmed using different experimental techniques in different cell types. ${ }^{10}$ The available evidence has been repetitively summarized in related review

Supported by the Deutsche Forschungsgemeinschaft grants AN372/ 23-1 and AN372/24-1.

Accepted for publication June 14, 2018.

Disclosures: None declared.

Address correspondence to Hans-Joachim Anders, M.D., Medizinische Klinik und Poliklinik IV, Klinikum der Universität München, Ziemssenstrasse 1, München 80336, Germany. E-mail: hjanders@med.unimuenchen.de 


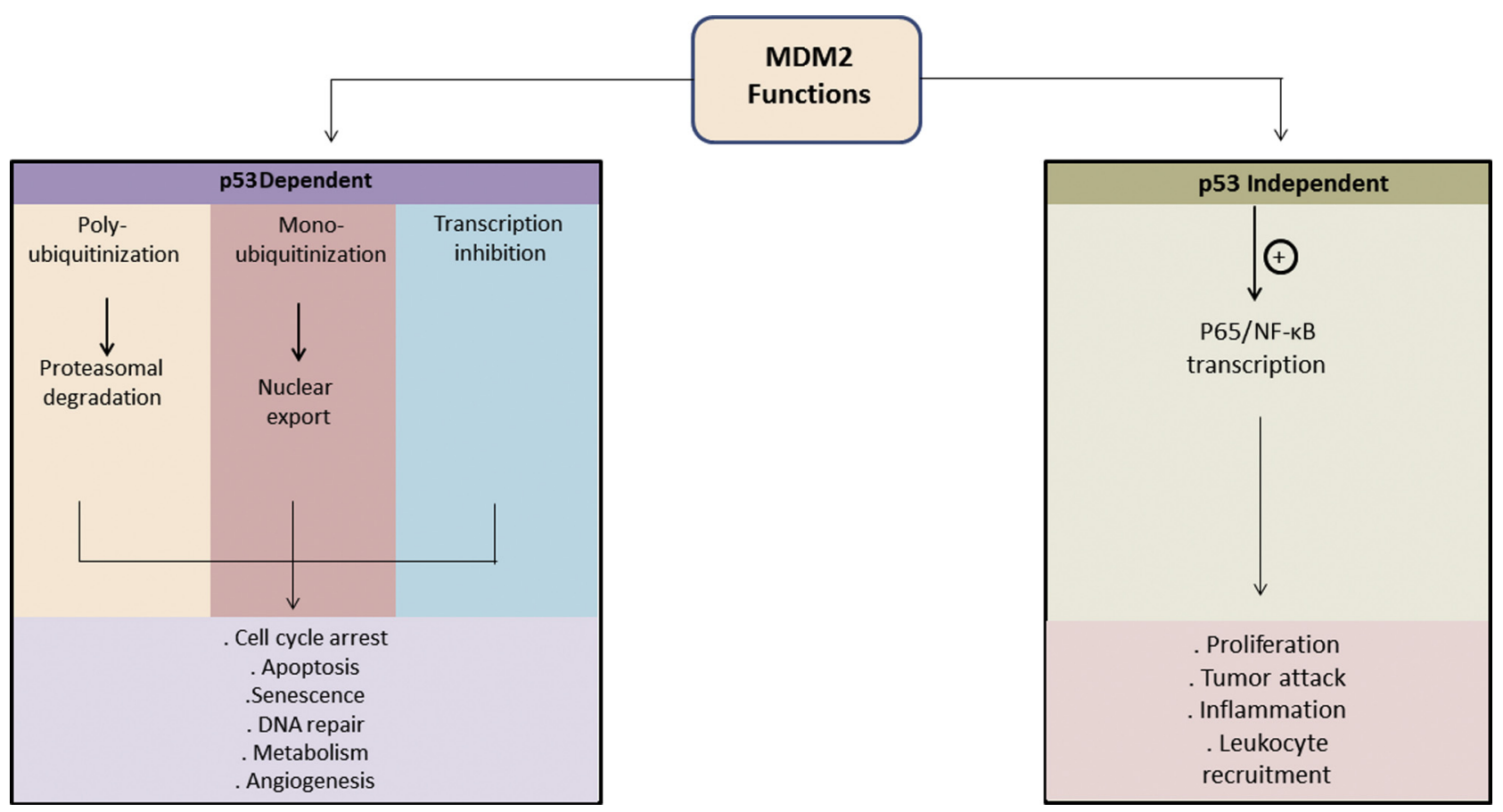

Figure 1 p53-Dependent and p53-independent functions of murine double minute (MDM)-2. MDM2 negatively regulates p53, as well as activates the NF- $\mathrm{B}$ signaling pathway.

articles. ${ }^{5,11}$ This NF- $\mathrm{B}$-agonistic role of MDM2 adds onto several other p53-independent functions of this protein. ${ }^{11,12}$

Therefore, as proposed by Fan et $\mathrm{al}^{8}$ for ocular inflammation, MDM2 blockade with drugs, such as Nutlin-3, could be an interesting alternative for the use of steroids in certain inflammatory disease states. ${ }^{13}$ Indeed, Mulay et $\mathrm{al}^{10}$ reported that Nutlin-3 can suppress the injury phase of postischemic acute kidney injury, a phase of the disease involving the autoamplification of cell necrosis and inflammation (ie, necroinflammation). ${ }^{14}$ Genetic p53 deficiency did not affect necroinflammation, and Nutlin-3 retained its suppressive effect, demonstrating a p53independent role for MDM2 in this early inflammatory phase of the disease. In the later phase, cell cycle activation is required to endorse compensatory hypertrophy of the surviving cells as well as progenitor-driven regeneration of lost tubular epithelial cells. ${ }^{15}$ Nutlin-3 blocked function and structure recovery of the kidney, a process entirely depending on the presence of $\mathrm{p} 53 .^{10}$ This study implied a bivalent function of MDM2 in acute inflammatory tissue injury versus subsequent repair, which raises caution about the right timing of therapeutic MDM2 inhibition. Indeed, studies with other disease models confirmed that the pathogenic role of MDM2 in disease is strictly context dependent. Restoring p53-driven cell cycle arrest and suppressing MDM2-driven inflammation can be useful in acute ischemic or toxic injuries to facilitate cell cycle arrest, thereby allowing DNA repair of injured cells with significant DNA damage. ${ }^{16}$ However, when cell cycle activation and cell proliferation is needed (eg, to replace injured cells after an acute injury), MDM2 blockade holds the risk to retard reepithelialization and wound healing. This risk profile is known from targeting the mammalian target of rapamycin. ${ }^{17}$ Indeed, mammalian target of rapamycin inhibitors have a similar risk profile because mammalian target of rapamycin and MDM2 share many biological functions in the context of inflammation versus regeneration in acute diseases.

But what exactly is the risk/benefit ratio of therapeutic MDM2 blockade in chronic disease states? From their shortterm data, Fan et $\mathrm{al}^{8}$ express great enthusiasm on a potential role of MDM2 inhibition in chronic uveitis, albeit no longterm outcome data from their disease model were presented. Long-term Nutlin-3 toxicity data were only provided in healthy animals. Can we be sure that MDM2 inhibition would not interfere with reparative processes in the eye? In chronic diseases, suppressing cell cycle activation for hypertrophy or proliferation with MDM2 inhibitors may outweigh the benefits of suppressing MDM2-driven inflammation. For example, long-term Nutlin-3 treatment of mice with type 2 diabetes aggravated diabetic kidney disease by affecting the capacity of tubular cells to adapt to the massively increased need for sodium and glucose reabsorption in the proximal tubule in diabetes. ${ }^{18}$ Synergistic effects of MDM2's p53-dependent and p53independent effects could be expected, in particular in diseases driven by inflammation and cell hyperplasia; hence, Nutlins were at first developed for malignancies. ${ }^{19}$ In addition, crescentic glomerulonephritis is a form of rapidly progressing kidney injury because of a massive inflammatory response in the renal glomeruli, together with 
hyperplasia of the parietal epithelial cells. Nutlin-3 treatment synergistically abrogated inflammation and epithelial hyperplasia and improved outcomes in mice with this disease. ${ }^{1}$ Another paradigmatic autoimmune disease characterized by hyperplasia of the lymphoid tissue is systemic lupus erythematosus. This polyclonal autoimmune disease is often complicated by inflammatory tissue injury [eg, of the kidneys (lupus nephritis) or of the skin (lupus rash)], arthritis, myositis, pneumonitis, or cerebritis. Nutlin-3 treatment had dramatic effects on MRLlpr/lpr mice with systemic lupus erythematosus-like disease (ie, a synergistic attenuation of the lymphoproliferative syndrome, subsequent autoantibody production, as well as lung and kidney inflammation and damage). ${ }^{20}$ The same might apply to other systemic autoimmune diseases, among those of chronic autoimmune uveitis. The data presented by Fan et al ${ }^{8}$ form a promising basis. Further studies using animal models mimicking the adaptive immunity-related pathogenesis of autoimmunity as well as a long-term outcome analysis of experimental uveitis will allow testing this concept.

\section{Supplemental Data}

Supplemental material for this article can be found at https://doi.org/10.1016/j.ajpath.2018.06.004.

\section{References}

1. Mulay SR, Romoli S, Desai J, Honarpisheh MM, Kumar SV, Anders HJ, Thomasova D: Murine double minute-2 inhibition ameliorates established crescentic glomerulonephritis. Am J Pathol 2016, 186:1442-1453

2. Shi D, Gu W: Dual roles of MDM2 in the regulation of p53: ubiquitination dependent and ubiquitination independent mechanisms of MDM2 repression of p53 activity. Genes Cancer 2012, 3:240-248

3. Zheng T, Wang J, Zhao Y, Zhang C, Lin M, Wang X, Yu H, Liu L, Feng Z, Hu W: Spliced MDM2 isoforms promote mutant p53 accumulation and gain-of-function in tumorigenesis. Nat Commun 2013, 4:2996

4. Clegg HV, Itahana K, Zhang Y: Unlocking the Mdm2-p53 loop: ubiquitin is the key. Cell Cycle 2008, 7:287-292

5. Ebrahim M, Mulay SR, Anders HJ, Thomasova D: MDM2 beyond cancer: podoptosis, development, inflammation, and tissue regeneration. Histol Histopathol 2015, 30:1271-1282
6. Eischen CM, Lozano G: The Mdm network and its regulation of p53 activities: a rheostat of cancer risk. Hum Mutat 2014, 35:728-737

7. Marine JC, Lozano G: Mdm2-mediated ubiquitylation: p53 and beyond. Cell Death Differ 2010, 17:93-102

8. Fan Y, Zhang W, Mahato B, Chavala SH: Inhibition of Noncanonical Mouse Double Minute 2 Homolog Abrogates Ocular Inflammation through NF-kB Suppression. Am J Pathol 2018, 188:2087-2097

9. Liu G, Park YJ, Tsuruta Y, Lorne E, Abraham E: p53 Attenuates lipopolysaccharide-induced NF-kappaB activation and acute lung injury. J Immunol 2009, 182:5063-5071

10. Mulay SR, Thomasova D, Ryu M, Anders HJ: MDM2 (murine double minute-2) links inflammation and tubular cell healing during acute kidney injury in mice. Kidney Int 2012, 81:1199-1211

11. Thomasova D, Mulay SR, Bruns H, Anders HJ: p53-Independent roles of MDM2 in NF-kappaB signaling: implications for cancer therapy, wound healing, and autoimmune diseases. Neoplasia 2012, 14: 1097-1101

12. Ganguli G, Wasylyk B: p53-Independent functions of MDM2. Mol Cancer Res 2003, 1:1027-1035

13. Gupta SC, Sundaram C, Reuter S, Aggarwal BB: Inhibiting NFkappaB activation by small molecules as a therapeutic strategy. Biochim Biophys Acta 2010, 1799:775-787

14. Mulay SR, Linkermann A, Anders HJ: Necroinflammation in kidney disease. J Am Soc Nephrol 2016, 27:27-39

15. Lazzeri E, Angelotti ML, Peired A, Conte C, Marschner JA, Maggi L, Mazzinghi B, Lombardi D, Melica ME, Nardi S, Ronconi E, Sisti A, Antonelli G, Becherucci F, De Chiara L, Guevara RR, Burger A, Schaefer B, Annunziato F, Anders HJ, Lasagni L, Romagnani P: Endocycle-related tubular cell hypertrophy and progenitor proliferation recover renal function after acute kidney injury. Nat Commun 2018, 9: 1344

16. Mulay SR, Thomasova D, Ryu M, Kulkarni OP, Migliorini A, Bruns H, Grobmayr R, Lazzeri E, Lasagni L, Liapis H, Romagnani P, Anders HJ: Podocyte loss involves MDM2-driven mitotic catastrophe. J Pathol 2013, 230:322-335

17. Tsang CK, Qi H, Liu LF, Zheng XF: Targeting mammalian target of rapamycin (mTOR) for health and diseases. Drug Discov Today 2007, $12: 112-124$

18. Saito R, Rocanin-Arjo A, You YH, Darshi M, Van Espen B, Miyamoto S, Pham J, Pu M, Romoli S, Natarajan L, Ju W, Kretzler M, Nelson R, Ono K, Thomasova D, Mulay SR, Ideker T, D'Agati V, Beyret E, Belmonte JC, Anders HJ, Sharma K: Systems biology analysis reveals role of MDM2 in diabetic nephropathy. JCI Insight 2016, 1:e87877

19. Impicciatore G, Sancilio S, Miscia S, Di Pietro R: Nutlins and ionizing radiation in cancer therapy. Curr Pharm Des 2010, 16:1427-1442

20. Allam R, Sayyed SG, Kulkarni OP, Lichtnekert J, Anders HJ: Mdm2 promotes systemic lupus erythematosus and lupus nephritis. J Am Soc Nephrol 2011, 22:2016-2027 